\title{
Asymmetric nucleophilic addition to vinylphosphonamidates (Part IV)
}

\author{
Kamyar Afarinkia,* Egizia De Pascale, and Sia Amara \\ Department of Chemistry, King's College London, London WC2R 2LS, UK. \\ E-mail: kamyar.afarinkia@kcl.ac.uk
}

\section{Dedicated to Professor Charles Rees on the occasion of his $75^{\text {th }}$ birthday}

(received 31 May 02; accepted 27 Sep 02; published on the web 05 Oct 02)

\begin{abstract}
Both stereoisomers of 6(R)-methyl-2-propenyl-1,3,2-oxazaphosphorinane-2-oxide 5a and 5b are prepared as single enantiomers. The configurations of the phosphorus atoms in the two compounds are deduced by NMR. The stereoselectivity in the addition of the phenyl carbanion to $\mathbf{5} \mathbf{a}$ and $\mathbf{5 b}$ are discussed.
\end{abstract}

Keywords: Vinylphosphonate, oxazaphosphorinane, cuprate, addition, stereoselective

\section{Introduction}

$\beta$-Substituted phosphonamidates and their phosphonate analogues are useful building blocks for the synthesis of many natural products. ${ }^{1}$ We have previously reported that these $\beta$-substituted phosphonamidates can be prepared diastereoselectively by the nucleophilic additions to asymmetric vinylphosphonamidates. ${ }^{2,3}$ For instance additions of a variety of carbon nucleophiles to chiral racemic 3-diphenylmethyl-2-(prop-2-enyl)-1,3,2-oxazaphosphorinane-2-oxide 1, proceed with good selectivities (typically $>80 \%$ de) and the relative configuration of the major diastereomers are $\left(\mathrm{S}_{\mathrm{P}}, \mathrm{S}_{\mathrm{C}(\beta)} / \mathrm{R}_{\mathrm{P}}, \mathrm{R}_{\mathrm{C}(\beta)}\right)$. Furthermore, the major isomer of these additions can be isolated after one crystallisation as a single diastereomer (Scheme 1). ${ }^{2}$

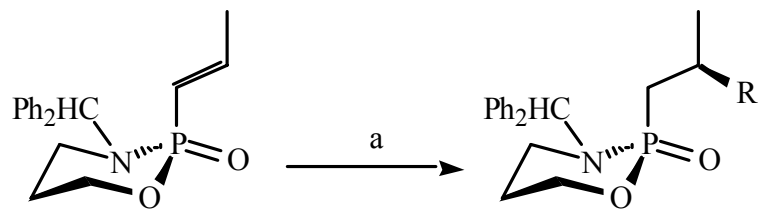

1

$\mathrm{R}=\mathrm{n}-\mathrm{C}_{4} \mathrm{H}_{9}, \mathrm{CH}_{2}=\mathrm{CH}, \mathrm{i}-\mathrm{C}_{3} \mathrm{H}_{7}, \mathrm{C}_{6} \mathrm{H}_{5}, \mathrm{t}-\mathrm{C}_{4} \mathrm{H}_{9}$; yield $13-65 \%$; d.e. $60-80 \%$

Scheme 1. $\operatorname{RMgBr}\left(6\right.$ eq), $\mathrm{CuI}\left(6\right.$ eq), $\mathrm{TMSCl}(6 \mathrm{eq}), \operatorname{TMEDA}(6 \mathrm{eq}),-78{ }^{\circ} \mathrm{C}$ to $-40{ }^{\circ} \mathrm{C}$, $\mathrm{Ar}$. 
We subsequently prepared both phosphorus antipodes of enantiomerically pure vinyl phosphonamidate 2a and $\mathbf{2 b}, \mathrm{R}_{\mathrm{P}}$ and $\mathrm{S}_{\mathrm{P}}$ 3-[( $\left.\mathrm{R}_{\mathrm{C}}\right)$-1-phenylethyl]-2-(prop-2-enyl)-2-oxo-1,3,2oxazaphosphorinane; and showed that the additions of carbon nucleophiles to these vinyl phosphonamidates also proceed diastereoselectively (Scheme 2). ${ }^{4}$

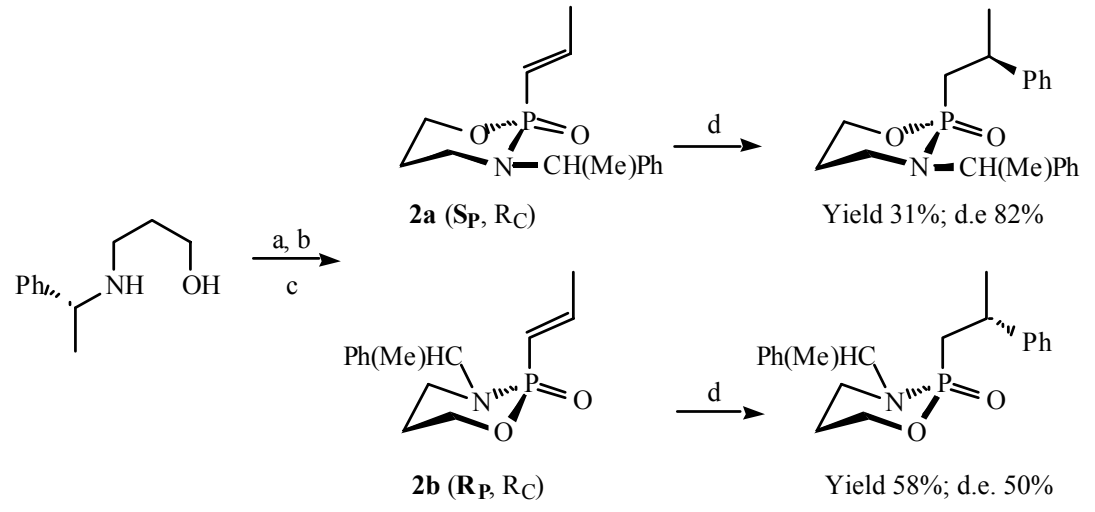

Scheme 2. (a) $\mathrm{EtOPCl}_{2}, \mathrm{Et}_{3} \mathrm{~N}, \mathrm{CH}_{2} \mathrm{Cl}_{2}$, reflux. (b) Allylbromide, reflux, $48 \mathrm{~h} ; 30 \%$ (over two steps). (c) $\mathrm{DBU}, \mathrm{CH}_{2} \mathrm{Cl}_{2}$, reflux, 5 days; 89\%. (d) $\mathrm{PhMgBr}$ (12 eq), CuI (6 eq), TMSCl (6 eq), TMEDA (6 eq), Et $\mathrm{E}_{2} \mathrm{O} / \mathrm{THF},-40{ }^{\circ} \mathrm{C}, 6 \mathrm{~h}$.

Therefore, we have a convenient method for the synthesis of enantiopure -substituted phosphonamidates starting from enantiopure vinyl phosphonamidates $\mathbf{2 a}$ and $\mathbf{2 b}$. However, development of alternative enantiopure vinyl phosphonamidates remains a key objective for us. This is because the origin of the chiral induction in the additions to $\mathbf{2 a}$ and $\mathbf{2} \mathbf{b}$ remains ambiguous since there are two asymmetric centers in the starting vinyl phosphonamidates. Investigation of the asymmetric addition to other vinyl phosphonamidate can help unravel some of the factors that are relevant in the process of these asymmetric inductions and can ultimately be used to further improve diastereoselection in these additions.

Our current model, which successfully predicts the sense of the diastereoselection in the additions to $\mathbf{2 a}$ and $\mathbf{2 b}$, suggests that in these compounds the chirality at the phosphorus atom alone is responsible for the chiral induction. 2 In other words, the chirality at the carbon atom on the nitrogen side chain has no influence on the chiral induction. To test if the selectivity would be affected if the chiral carbon atom was at a different position. Therefore, we decided to study the additions of carbon nucleophiles to both phosphorus antipodes of enantiomerically pure vinyl phosphonamidate $\mathbf{4 a}$ and $\mathbf{4 b}, \mathrm{R}_{\mathrm{P}}$ and $\mathrm{S}_{\mathrm{P}}$ 6(R)-methyl-2-propenyl-1,3,2-oxazaphosphorinane-2oxide.

\section{Results and Discussion}

Diastereomeric allyl phosphonamidates $4 \mathbf{a}$ and $\mathbf{4 b}$ were prepared in two steps from N(phenylmethyl)-1(R)-methylpropanolamine $\mathbf{3}^{2,4}$ itself prepared from methyl (R)-3- 
hydroxybutyrate in two steps (Scheme 3). The two diastereomers were cleanly separated by chromatography. Unfortunately neither of the materials was crystalline and we were unable to confirm the absolute configuration of the phosphorus atom in these molecules. However, we were able to infer the configuration of the phosphorus atom from NOESY NMR data. In compound $\mathbf{4 b}$, the side chain methylene protons attached to the phosphorus atom show no n.O.e. enhancement with H-6 nor H-4ax. However, in compound 4a, the side chain methylene protons show strong n.O.e. enhancement with $\mathrm{H}-6$ and $\mathrm{H}-4_{\mathrm{ax}}$. This would be consistent with the proposed structure for compounds $\mathbf{4 a}$ and $\mathbf{4 b}$ (Scheme 3).

Both diastereomers were then individually transformed to the corresponding vinyl phosphonamidates 5a and 5b (Scheme 4). ${ }^{5}$ Here the NOESY NMR data also confirm our assignment. In compound $\mathbf{5 b}$, the olefinic proton at the position $\alpha$ to the phosphorus atom shows no n.O.e. enhancement with H-6 nor H-4ax. However, in compound 5a, the same proton shows strong n.O.e. enhancement with $\mathrm{H}-6$ and $\mathrm{H}-4_{\mathrm{ax}}$.

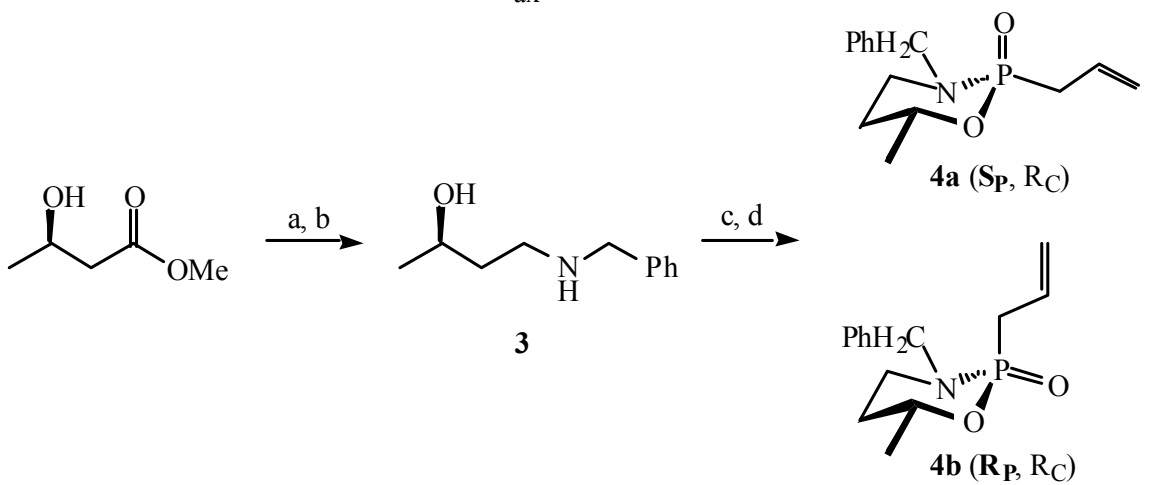

Scheme 3. (a) $\mathrm{PhCH}_{2} \mathrm{NH}_{2} \mathrm{MeOH}$, reflux, 5 days; 90\%. (b) $\mathrm{LiAlH}_{4}, \mathrm{THF} ; 68 \%$. (c) $\mathrm{EtOPCl}_{2}$, $\mathrm{Et}_{3} \mathrm{~N}, \mathrm{CH}_{2} \mathrm{Cl}_{2}$, reflux. (d) Allylbromide, reflux, $48 \mathrm{~h} ; 39 \%$ (over two steps).

We then carried out the reactions of phenylcuprates in the presence of $\mathrm{TMSCl}$ (chlorotrimethylsilane) and TMEDA (N,N,N',N'-tetramethylethylenediamine) as rateaccelerating additives. ${ }^{5}$ The results are outlined below (Scheme 4 ).

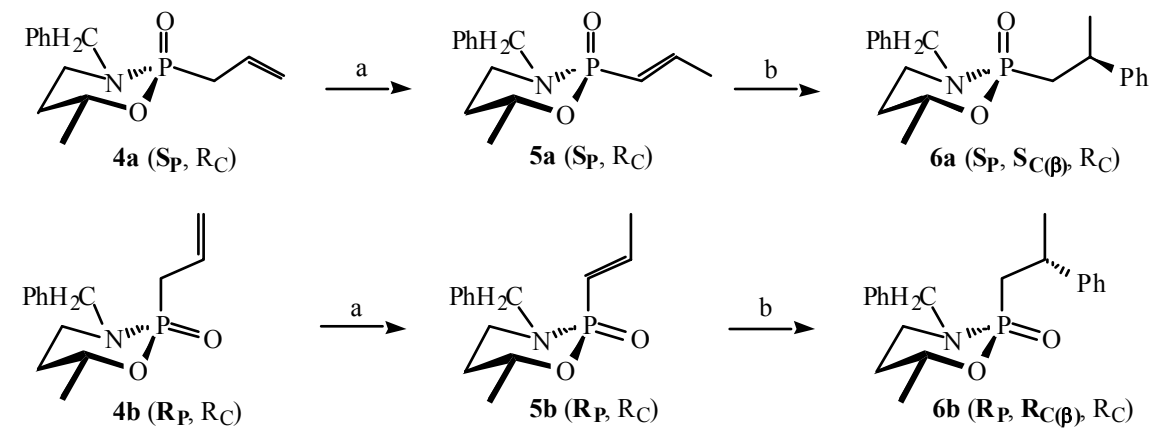

Scheme 4. (a) DBU, $\mathrm{CH}_{2} \mathrm{Cl}_{2}$, reflux, 5 days; 71\%. (b) $\mathrm{PhMgBr}$ (6 eq), $\mathrm{CuI}$ (6 eq), TMSCl (6 eq), TMEDA (6 eq), $\mathrm{Et}_{2} \mathrm{O} / \mathrm{THF},-40^{\circ} \mathrm{C}, 6 \mathrm{~h}$. 
${ }^{31} \mathrm{P}$ NMR analysis of the crude reaction mixture from the addition of the phenyl anion to $\mathbf{5 a}$, which has an $\mathrm{S}$ configuration at the phosphorus atom, showed a 5:1 ratio in favour of the higher ppm stereoisomer. That result is consistent with a 10:1 ratio in favour of the higher ppm stereoisomer observed in the addition of the phenyl anion to $\mathbf{2 a}$, which also has an $\mathrm{S}$ configuration at the phosphorus atom. Similarly, analysis of the crude reaction mixture from addition of the phenyl anion to $\mathbf{5 b}$, which has an $\mathrm{R}$ configuration at the phosphorus atom, showed a 4:1 ratio in favour of the higher ppm stereoisomer. This result is consistent with a 3:1 ratio in favour of the higher ppm stereoisomer observed in the addition of the phenyl anion to $\mathbf{2} \mathbf{b}$, which also has an $\mathrm{R}$ configuration at the phosphorus atom. We can conclude that in the addition of the phenyl anion, the sense and magnitude of chiral induction is similar between 2a and 5a and between $\mathbf{2 b}$ and $\mathbf{5 b}$.

In conclusion, vinyl phosphonamidates $\mathbf{5 a}$ and $\mathbf{5 b}$ are useful alternative vinyl phosphonamidate precursors for the synthesis of enantiomercially pure $\beta$-substituted phosphoamidates. Furthermore, the similarity in the magnitude and the sense of selectivity in the additions of the phenyl anion suggests that the chiral induction in the additions to $\mathbf{2} \mathbf{a}$ or $\mathbf{2} \mathbf{b}$ and $\mathbf{5 a}$ or $\mathbf{5 b}$, is determined primarily by the chirality at the phosphorus atom as previously proposed (Scheme 5). ${ }^{2}$ This confirms our working model which rationalises the observed selectivities based on a combination of steric and internal chelation effects.

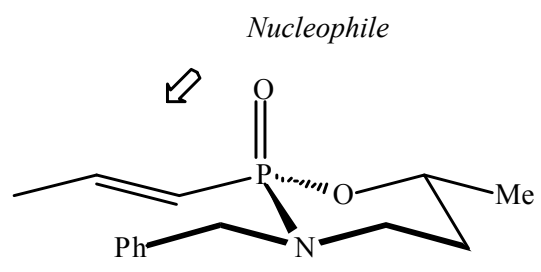

Addition to 5a

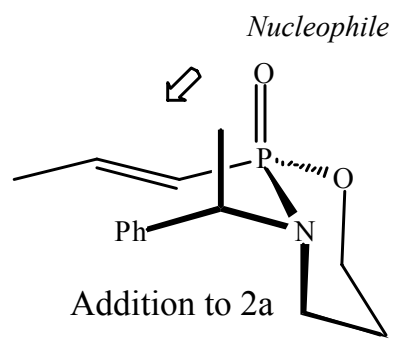

Scheme 5. Diastereofacial selectivity in the additions to $\mathbf{2 a}$ and $\mathbf{5 a}$.

\section{Experimental Section}

General Procedures. NMR spectra were obtained in $\mathrm{CDCl}_{3}$ (unless specified) on Bruker AMX360 and AMX400 spectrometers operating respectively at 360 and $400 \mathrm{MHz}$ for ${ }^{1} \mathrm{H}, 90.6$ and $101 \mathrm{MHz}$ for ${ }^{13} \mathrm{C}$ and 146 and $162 \mathrm{MHz}$ for ${ }^{31} \mathrm{P}$. Chemical shifts are reported in ppm. Assignments of NMR spectra are confirmed by COSY, NOSEY, DEPT techniques and C-H correlation spectroscopy where necessary. Mass spectra were obtained on a Jeol AX505W mass spectrometer using electron impact (EI) and chemical ionisation (CI) techniques. Infra-red spectra were recorded on a Perkin-Elmer 1600 FTIR instrument. Chromatography was carried out on silica gel (Merck 9385). Petroleum ether used throughout is boiling point fraction $60-80{ }^{\circ} \mathrm{C}$. 
$\boldsymbol{N}$-Benzyl- $\boldsymbol{N}$-[3(R)-hydroxybutyl]amine (3). A solution of methyl (R)-3-hydroxybutyrate ${ }^{6}$ $(30.0 \mathrm{~g}, 0.254 \mathrm{mmol})$ and benzylamine $(27.2 \mathrm{~g}, 0.254 \mathrm{mmol})$ in methanol $(15 \mathrm{~mL})$ was refluxed for 5 days. The reaction was analysed by $\mathrm{nmr}$ to confirm the complete formation of the ester. Solvent was evaporated and the residue was recrystallised from ethylacetate to afford $N$-benzyl3(R)-hydroxybutyramide as a white solid (26.7 g first crop, $12.1 \mathrm{~g}$ second crop, $5.6 \mathrm{~g}$ third crop, $90 \%$ total). Mp. $79-80{ }^{\circ} \mathrm{C},[\alpha]_{\mathrm{D}}=-15.7^{\circ}\left(\mathrm{c}=0.24\right.$, ethanol); ${ }^{1} \mathrm{H}$ NMR $\left(\mathrm{CDCl}_{3}\right): \delta 1.21(3 \mathrm{H}, \mathrm{d}, \mathrm{J}$ $\left.=7 \mathrm{~Hz}, \underline{\mathrm{C}}_{3} \mathrm{CH}\right), 2.28(1 \mathrm{H}, \mathrm{dd}, \mathrm{J}=10 \mathrm{~Hz}, 17 \mathrm{~Hz}, \mathrm{C} \underline{\mathrm{HH}}), 2.37(1 \mathrm{H}, \mathrm{dd}, \mathrm{J}=3 \mathrm{~Hz}, 17 \mathrm{~Hz}, \mathrm{CH} \underline{\mathrm{H}})$, $3.89(1 \mathrm{H}, \mathrm{bs}, \mathrm{OH}), 4.18(1 \mathrm{H}, \mathrm{m}, \mathrm{MeC} \underline{\mathrm{HO}}), 4.42\left(2 \mathrm{H}, \mathrm{d}, \mathrm{J}=6 \mathrm{~Hz}, \mathrm{CH}_{2} \mathrm{~N}\right), 6.42(1 \mathrm{H}, \mathrm{bs}, \mathrm{NH})$, 7.27-7.35 (5H, m, aromatic H). ${ }^{13} \mathrm{C} \mathrm{NMR}\left(\mathrm{CDCl}_{3}\right): \delta 22.87(\mathrm{CH} 3), 43.37\left(\mathrm{CH}_{2} \mathrm{CO}\right), 43.86$ $\left(\mathrm{CH}_{2} \mathrm{Ph}\right), 64.86(\mathrm{MeC} \underline{\mathrm{HO}}), 127.55$ (aromatic $\left.\mathrm{CH}\right), 127.72(2 \mathrm{x}$ aromatic $\mathrm{CH}), 128.74(2 \mathrm{x}$ aromatic $\mathrm{CH}$ ), 137.97 (aromatic C), 172.28 (으); m/z (CI, ammonia) $387\left(\mathrm{M}_{2} \mathrm{H}^{+}, 45\right), 211$ $\left(\mathrm{MNH}_{4}{ }^{+}, 61\right), 194\left(\mathrm{MH}^{+}, 100\right)$; HRMS calc for $\mathrm{C}_{11} \mathrm{H}_{16} \mathrm{NO}_{2}\left(\mathrm{MH}^{+}\right)$194.1181, found 194.1151. NBenzyl-3(R)-hydroxybutyramide $(24.18 \mathrm{~g}, 0.125 \mathrm{mmol})$ was added to a cooled suspension of lithium aluminium hydride $(10 \mathrm{~g}, 0.263 \mathrm{mmol})$ in THF $(250 \mathrm{~mL})$ over 30 minutes. The mixture was refluxed for 3 hours and then quenched by sequential slow addition of water $(10 \mathrm{~mL}), 15 \%$ aqueous sodium hydroxide $(10 \mathrm{~mL})$ and water $(27 \mathrm{~mL})$. Solid was filterd off and the filterate was stripped of solvent in vaccu to afford the title compound as a pale yellow oil $(15.31 \mathrm{~g}, 68 \%)$. $[\alpha]_{\mathrm{D}}=+1.8^{\circ}(\mathrm{c}=0.5$, ethanol $) ;{ }^{1} \mathrm{H} \mathrm{NMR}\left(\mathrm{CDCl}_{3}\right): \delta 1.17\left(3 \mathrm{H}, \mathrm{d}, \mathrm{J}=6 \mathrm{~Hz}, \mathrm{CH}_{3} \mathrm{CH}\right), 1.51-1.62$ $\left(2 \mathrm{H}, \mathrm{m}, \mathrm{CCH}_{2} \mathrm{C}\right), 2.78(1 \mathrm{H}, \mathrm{ddd}, \mathrm{J}=4.5 \mathrm{~Hz}, 9.5 \mathrm{~Hz}, 11 \mathrm{~Hz}, \mathrm{CH} \underline{\mathrm{HN}}), 3.00$ (1H, ddd, J = $4 \mathrm{~Hz}, 4.5$ Hz, $22 \mathrm{~Hz}, \mathrm{C} \underline{H} H \mathrm{~N}), 3.74(1 \mathrm{H}, \mathrm{d}, \mathrm{J}=13 \mathrm{~Hz}, \mathrm{CH} \underline{H P h}), 3.82(1 \mathrm{H}, \mathrm{d}, \mathrm{J}=13 \mathrm{~Hz}, \mathrm{C} \underline{H} H \mathrm{Ph}), 3.98$ (1H, $\mathrm{m}, \mathrm{MeC} \underline{\mathrm{HO}}), 7.23-7.28(5 \mathrm{H}, \mathrm{m}$, aromatic $\mathrm{H}) .{ }^{13} \mathrm{C}\{\mathrm{H}\} \mathrm{NMR}\left(\mathrm{CDCl}_{3}\right): \delta 23.51\left(\mathrm{CH}_{3}\right), 36.7$ $\left(\mathrm{CCH}_{2} \mathrm{C}\right), 48.20\left(\mathrm{NCH}_{2}\right), 53.77\left(\mathrm{CH}_{2} \mathrm{Ph}\right), 69.54(\mathrm{Me} \underline{\mathrm{CHO}}), 127.17$ (aromatic $\left.\mathrm{CH}\right), 128.15(2 \mathrm{x}$ aromatic $\mathrm{CH}$ ), 128.48 (2 x aromatic $\mathrm{CH}$ ), 139.38 (aromatic $\mathrm{C}$ ); m/z (CI, ammonia) $359\left(\mathrm{M}_{2} \mathrm{H}^{+}\right.$, 20), $180\left(\mathrm{MH}^{+}, 100\right)$; HRMS calc for $\mathrm{C}_{11} \mathrm{H}_{18} \mathrm{NO}\left(\mathrm{MH}^{+}\right)$180.1388, found 180.1360 .

(6R,2R) 6-Methyl-3-benzyl-2-(prop-2'-enyl)-1,3,2-oxazaphosphorinane-2-oxide (4a) and (6R,2S) 6-Methyl-3-benzyl-2-(prop-2'-enyl)-1,3,2-oxazaphosphorinane-2-oxide (4b).

Triethylamine $(6.80 \mathrm{~mL}, 49.0 \mathrm{mmol})$ was added to a refluxing solution of ethyldichlorophosphite (3.608 g, $24.5 \mathrm{mmol})$ in DCM $(50 \mathrm{~mL})$ maintained under a nitrogen atmosphere. The solution was stirred for 10 minutes before addition of N-Benzyl-N-[3(R)-hydroxybutyl]amine (4.395 g, $24.5 \mathrm{mmol})$ as a solution in DCM $(20 \mathrm{~mL})$. The stirring was continued overnight. The solvent was evaporated, diethylether $(50 \mathrm{~mL})$ was added and the mixture was filtered through celite under a blanket of nitrogen gas. The filterate was evaporated to afford 2-ethoxy-6-methyl-3benzyl-1,3,2-oxazaphosphorinane as a sticky oil. This was dissolved in a large excess of allyl bromide $(21.5 \mathrm{~mL})$ and refluxed under an argon atmosphere for 1.5 days. Evaporation of solvent followed by chromatography using ethyl acetate as eluent afforded (6R,2R) 6-Methyl-3-benzyl-2(prop-2'-enyl)-1,3,2-oxazaphosphorinane-2-oxide as an oil (1.52 g, 23\%). $\mathrm{R}_{\mathrm{f}} 0.25$ (ethyl acetate). ${ }^{1} \mathrm{H}$ NMR $\left(\mathrm{CDCl}_{3}\right): \delta 1.36\left(3 \mathrm{H}, \mathrm{dd}, \mathrm{J}_{\mathrm{H}}=7 \mathrm{~Hz}, \mathrm{~J}_{\mathrm{P}}=2 \mathrm{~Hz}, \mathrm{CH}_{3}\right), 1.64-1.78\left(2 \mathrm{H}, \mathrm{m}, \mathrm{CH}_{2} \mathrm{C}_{2} \mathrm{CH}_{2}\right)$, $2.76\left(2 \mathrm{H}, \mathrm{ddd}, \mathrm{J}_{\mathrm{H}}=1,7 \mathrm{~Hz}, \mathrm{~J}_{\mathrm{P}}=19 \mathrm{~Hz}, \mathrm{PCH}_{2}\right), 2.92-3.12\left(2 \mathrm{H}, \mathrm{m}, \mathrm{CH}_{2} \mathrm{~N}\right), 3.85\left(1 \mathrm{H}, \mathrm{dd}, \mathrm{J}_{\mathrm{H}}=15\right.$ $\left.\mathrm{Hz}, \mathrm{J}_{\mathrm{P}}=7 \mathrm{~Hz}, \mathrm{PhC} \underline{\mathrm{HH}}\right), 4.42(1 \mathrm{H}, \mathrm{m}, \mathrm{C} \underline{\mathrm{HMeO}}), 4.48\left(1 \mathrm{H}, \mathrm{dd}, \mathrm{J}_{\mathrm{H}}=15 \mathrm{~Hz}, \mathrm{~J}_{\mathrm{P}}=7 \mathrm{~Hz}, \mathrm{PhCH} \underline{\mathrm{H}}\right)$, 5.13-5.26 (2H, m, $\left.\mathrm{CH}_{2}=\right), 5.90(\mathrm{~m}, \mathrm{CH}=), 7.21-7.58(5 \mathrm{H}, \mathrm{m}$, aromatic $\mathrm{H}) .{ }^{31} \mathrm{P}\{\mathrm{H}\} \mathrm{NMR}\left(\mathrm{CDCl}_{3}\right)$ : 
$\delta$ 24.62. ${ }^{13} \mathrm{C}\{\mathrm{H}\} \mathrm{NMR}\left(\mathrm{CDCl}_{3}\right): \delta 22.97\left(\mathrm{~d}, \mathrm{~J}_{\mathrm{P}}=7 \mathrm{~Hz}, \mathrm{Me}\right), 31.16\left(\mathrm{~d}, \mathrm{~J}_{\mathrm{P}}=119 \mathrm{~Hz}, \mathrm{PCH}_{2}\right), 33.88$ $\left(\mathrm{d}, \mathrm{J}_{\mathrm{P}}=5 \mathrm{~Hz}, \mathrm{C}-5\right), 45.27\left(\mathrm{~d}, \mathrm{~J}_{\mathrm{P}}=2 \mathrm{~Hz}, \mathrm{CH}_{2} \mathrm{~N}\right), 51.72\left(\mathrm{~d}, \mathrm{~J}_{\mathrm{P}}=4 \mathrm{~Hz}, \mathrm{CH}_{2} \mathrm{Ph}\right), 76.87\left(\mathrm{~d}, \mathrm{~J}_{\mathrm{P}}=7 \mathrm{~Hz}\right.$, $\underline{\mathrm{CHMeO}}, 118.81\left(\mathrm{~d}, \mathrm{~J}_{\mathrm{P}}=13 \mathrm{~Hz}, \mathrm{CH}_{2}=\right), 127.83$ (aromatic $\mathrm{CH}$ ), $128.8(2 \mathrm{x}$ aromatic $\mathrm{CH}), 129.05$ $\left(\mathrm{d}, \mathrm{J}_{\mathrm{P}}=10 \mathrm{~Hz},-\mathrm{CH}=\right), 138.12\left(\mathrm{~d}, \mathrm{~J}_{\mathrm{P}}=5 \mathrm{~Hz}\right.$, aromatic $\left.\mathrm{C}\right) ; \mathrm{m} / \mathrm{z}\left(\mathrm{CI}\right.$, ammonia) $283\left(\mathrm{MNH}_{4}{ }^{+}, 61\right)$, $266\left(\mathrm{MH}^{+}, 14\right), 254$ (100); HRMS calc for $\mathrm{C}_{14} \mathrm{H}_{20} \mathrm{NO}_{2} \mathrm{P}\left(\mathrm{MH}^{+}\right)$266.1310, found 266.1303. Further elution of the chromatography coloumn with a gradient of $2-5 \% \mathrm{v} / \mathrm{v}$ methanol in ethylacetate afforded (6R,2S) 6-Methyl-3-benzyl-2-(prop-2'-enyl)-1,3,2-oxazaphosphorinane-2oxide as an oil $(1.05 \mathrm{~g}, 16 \%) . \mathrm{R}_{\mathrm{f}} 0.05$ (ethyl acetate). ${ }^{1} \mathrm{H} \mathrm{NMR}\left(\mathrm{CDCl}_{3}\right): \delta 1.32\left(3 \mathrm{H}, \mathrm{dd}, \mathrm{J}_{\mathrm{H}}=6\right.$ $\left.\mathrm{Hz}, \mathrm{J}_{\mathrm{P}}=2 \mathrm{~Hz}, \mathrm{CH}_{3}\right), 1.64-1.79\left(2 \mathrm{H}, \mathrm{m}, \mathrm{CH}_{2} \underline{\mathrm{C}}_{2} \mathrm{CH}_{2}\right), 2.73\left(2 \mathrm{H}, \mathrm{dd}, \mathrm{J}_{\mathrm{H}}=7 \mathrm{~Hz}, \mathrm{~J}_{\mathrm{P}}=21 \mathrm{~Hz}\right.$, $\left.\mathrm{PCH}_{2}\right), 2.89-3.11\left(2 \mathrm{H}, \mathrm{m}, \mathrm{CH}_{2} \mathrm{~N}\right), 3.85\left(2 \mathrm{H}, \mathrm{m}, \mathrm{PhCH}_{2}\right), 4.57(1 \mathrm{H}, \mathrm{m}, \mathrm{C} \underline{\mathrm{HMeO}}), 5.11-5.23(2 \mathrm{H}$, $\left.\mathrm{m}, \mathrm{CH}_{2}=\right)$, $5.81(\mathrm{~m}, \mathrm{CH}=), 7.25-7.55(5 \mathrm{H}, \mathrm{m}$, aromatic $\mathrm{H}) .{ }^{31} \mathrm{P}\{\mathrm{H}\} \mathrm{NMR}\left(\mathrm{CDCl}_{3}\right): \delta 28.34$. ${ }^{13} \mathrm{C}\{\mathrm{H}\}$ NMR $\left(\mathrm{CDCl}_{3}\right): \delta 22.56\left(\mathrm{~d}, \mathrm{~J}_{\mathrm{P}}=8 \mathrm{~Hz}, \mathrm{Me}\right), 33.28\left(\mathrm{~d}, \mathrm{~J}_{\mathrm{P}}=3 \mathrm{~Hz}, \mathrm{C}-5\right), 34.92\left(\mathrm{~d}, \mathrm{~J}_{\mathrm{P}}=131\right.$ $\left.\mathrm{Hz}, \mathrm{PCH}_{2}\right), 45.29\left(\mathrm{CH}_{2} \mathrm{~N}\right), 52.12\left(\mathrm{~d}, \mathrm{~J}_{\mathrm{P}}=4 \mathrm{~Hz}, \mathrm{CH}_{2} \mathrm{Ph}\right), 73.25\left(\mathrm{~d}, \mathrm{~J}_{\mathrm{P}}=8 \mathrm{~Hz}, \underline{\mathrm{CHMeO}}\right), 119.65$ $\left(\mathrm{d}, \mathrm{J}_{\mathrm{P}}=14 \mathrm{~Hz}, \mathrm{CH}_{2}=\right), 127.86$ (aromatic $\mathrm{CH}$ ), 128.74 (aromatic $\mathrm{CH}$ ), 128.91 (aromatic $\mathrm{CH}$ ), $129.40\left(\mathrm{~d}, \mathrm{~J}_{\mathrm{P}}=10 \mathrm{~Hz},-\mathrm{CH}=\right), 137.81\left(\mathrm{~d}, \mathrm{~J}_{\mathrm{P}}=5 \mathrm{~Hz}\right.$, aromatic $\left.\mathrm{C}\right) ; \mathrm{m} / \mathrm{z}$ (CI, ammonia) 283 $\left(\mathrm{MNH}_{4}{ }^{+}, 61\right), 266\left(\mathrm{MH}^{+}, 14\right), 254$ (100); HRMS calc for $\mathrm{C}_{14} \mathrm{H}_{20} \mathrm{NO}_{2} \mathrm{P}\left(\mathrm{MH}^{+}\right)$266.1310, found 266.1299.

(6R,2S) 6-Methyl-3-benzyl-2-(prop-1'-enyl)-1,3,2-oxazaphosphorinane-2-oxide (5b). A solution of DBU (0.91 mL, $5.9 \mathrm{mmol})$ and (6R,2S) 6-Methyl-3-benzyl-2-(prop-2'-enyl)-1,3,2oxazaphosphorinane-2-oxide (1.05 g, $3.96 \mathrm{mmol} \mathrm{mmol})$ in DCM $(5 \mathrm{~mL})$ was heated at reflux under argon atmosphere for 36 hours. The solvent was then evaporated and the product was purified by chromatographic column using ethyl acetate as eluent to afford the title compound as an oil $\left(0.75 \mathrm{~g} \mathrm{71 \% ).}{ }^{1} \mathrm{H}\right.$ NMR $\left(\mathrm{CDCl}_{3}\right): \delta 1.35\left(3 \mathrm{H}, \mathrm{dd}, \mathrm{J}_{\mathrm{H}}=6 \mathrm{~Hz}, \mathrm{~J}_{\mathrm{P}}=1 \mathrm{~Hz}, \mathrm{CH}_{3}-\mathrm{C} 6\right), 1.71-1.79$ $\left(2 \mathrm{H}, \mathrm{m}, \mathrm{CH}_{2} \mathrm{C}_{2} \mathrm{CH}_{2}\right), 1.95\left(3 \mathrm{H}, \mathrm{dd}, \mathrm{J}_{\mathrm{H}}=2 \mathrm{~Hz}, \mathrm{~J}_{\mathrm{P}}=4 \mathrm{~Hz}, \mathrm{CH}_{3} \mathrm{CH}=\right), 2.85-3.13\left(2 \mathrm{H}, \mathrm{m}, \mathrm{CH}_{2} \mathrm{~N}\right)$, $3.89\left(1 \mathrm{H}, \mathrm{dd}, \mathrm{J}_{\mathrm{H}}=15 \mathrm{~Hz}, \mathrm{~J}_{\mathrm{P}}=4 \mathrm{~Hz}, \mathrm{PhC} \underline{\mathrm{HH}}\right), 4.23\left(1 \mathrm{H}, \mathrm{dd}, \mathrm{J}_{\mathrm{H}}=15 \mathrm{~Hz}, \mathrm{~J}_{\mathrm{P}}=10 \mathrm{~Hz}, \mathrm{PhC} \underline{H} \mathrm{H}\right)$, $4.65(1 \mathrm{H}, \mathrm{m}, \mathrm{C} \underline{\mathrm{HMeO}}), 5.73\left(1 \mathrm{H}, \mathrm{ddd}, \mathrm{J}_{\mathrm{H}}=2,17 \mathrm{~Hz}, \mathrm{~J}_{\mathrm{P}}=19 \mathrm{~Hz}, \mathrm{MeCH}=\right), 6.90\left(1 \mathrm{H}, \mathrm{ddd}, \mathrm{J}_{\mathrm{H}}=\right.$ 7,17 Hz, $\left.\mathrm{J}_{\mathrm{P}}=21 \mathrm{~Hz}, \mathrm{PCH}=\right), 7.24-7.37(5 \mathrm{H}, \mathrm{m}$, aromatic $\mathrm{H}) .{ }^{31} \mathrm{P}\{\mathrm{H}\} \mathrm{NMR}\left(\mathrm{CDCl}_{3}\right): \delta 20.70$. ${ }^{13} \mathrm{C}\{\mathrm{H}\}$ NMR $\left(\mathrm{CDCl}_{3}\right): \delta 20.08\left(\mathrm{~d}, \mathrm{~J}_{\mathrm{P}}=23 \mathrm{~Hz}, \mathrm{CH}_{3} \mathrm{CH}=\right), 22.39\left(\mathrm{~d}, \mathrm{~J}_{\mathrm{P}}=9 \mathrm{~Hz}, \underline{\mathrm{CH}}_{3}-\mathrm{C} 6\right), 33.26$ $\left(\mathrm{d}, \mathrm{J}_{\mathrm{P}}=3 \mathrm{~Hz}, \mathrm{C}-5\right), 44.42\left(\mathrm{CH}_{2} \mathrm{~N}\right), 51.22\left(\mathrm{~d}, \mathrm{~J}_{\mathrm{P}}=5 \mathrm{~Hz}, \mathrm{CH}_{2} \mathrm{Ph}\right), 72.64\left(\mathrm{~d}, \mathrm{~J}_{\mathrm{P}}=6 \mathrm{~Hz}, \underline{\mathrm{CHMeO}}\right)$, $121.17\left(\mathrm{~d}, \mathrm{~J}_{\mathrm{P}}=180 \mathrm{~Hz}, \mathrm{PCH}=\right), 127.37$ (aromatic $\left.\mathrm{CH}\right), 128.13$ (aromatic $\left.\mathrm{CH}\right), 128.48$ (aromatic $\mathrm{CH}), 137.63\left(\mathrm{~d}, \mathrm{~J}_{\mathrm{P}}=8 \mathrm{~Hz}\right.$, aromatic $\left.\mathrm{C}\right), 150.25\left(\mathrm{~d}, \mathrm{~J}_{\mathrm{P}}=5 \mathrm{~Hz}, \mathrm{MeCH}=\right) ; \mathrm{m} / \mathrm{z}(\mathrm{EI}) 266\left(\mathrm{MH}^{+}, 54\right)$, 225 (12), 175 (100); HRMS calc for $\mathrm{C}_{14} \mathrm{H}_{20} \mathrm{NO}_{2} \mathrm{P}\left(\mathrm{MH}^{+}\right)$266.1310, found 266.1259.

(6R,2S, 2'S) 6-Methyl-3-benzyl-2-(2'-phenylpropyl)-1,3,2-oxazaphosphorinane-2-oxide (6b). Phenyl magnesium bromide (3M solution in diethylether, $3.0 \mathrm{~mL}, 9.00 \mathrm{mmol})$ was added to a stirred suspension of $\mathrm{CuI}(0.86 \mathrm{~g}, 4.53 \mathrm{mmol})$ in dry diethyl ether $(7.8 \mathrm{~mL})$, maintained under a dry argon atmosphere at $-40{ }^{\circ} \mathrm{C}$. After 45 minutes, the solution was cooled to $-78{ }^{\circ} \mathrm{C}$ and $\mathrm{TMSCl}$ $(0.57 \mathrm{~mL}, 4.53 \mathrm{mmol})$ was added followed after $10 \mathrm{~min}$. by TMEDA $(0.68 \mathrm{~mL}, 4.53 \mathrm{mmol})$ and then a solution of (6R,2S) 6-Methyl-3-benzyl-2-(prop-1'-enyl)-1,3,2-oxazaphosphorinane-2oxide $5 \mathbf{b}(0.20 \mathrm{~g}, 0.075 \mathrm{mmol})$ in dry THF $(7.8 \mathrm{ml})$. Reaction was monitored by tlc (EtOAc as eluant) and was found to be complete after 5 hours. The reaction mixture was quenched by 
addition of sat. aqueous $\mathrm{NH}_{4} \mathrm{Cl}(50 \mathrm{~mL})$ and filtered through Celite ${ }^{\circledR}$. Solid was washed with ethylacetate $(50 \mathrm{~mL})$. The organic and aqueous phases of the filtrate were separated and the aqueous phase was washed with ethyl acetate $(3 \times 30 \mathrm{~mL})$. The combined organic extracts were washed with brine and then dried over $\mathrm{MgSO}_{4}$. Evaporation of solvent afforded a green oil. The crude product was directly chromatographed on silica gel (Merck 9385) using EtOAc as eluent to afford the title compound as an oil (180 mg, 69\%). A fraction containing unreacted starting material was also isolated $(20 \mathrm{mg}) .{ }^{1} \mathrm{H} \mathrm{NMR}\left(\mathrm{CDCl}_{3}\right): \delta 1.20\left(3 \mathrm{H}, \mathrm{dd}, \mathrm{J}_{\mathrm{H}}=7 \mathrm{~Hz}, \mathrm{~J}_{\mathrm{P}}=1 \mathrm{~Hz}\right.$, $\left.\mathrm{C}_{3} \mathrm{CHPh}\right), 1.37\left(3 \mathrm{H}, \mathrm{d}, \mathrm{J}_{\mathrm{H}}=6 \mathrm{~Hz}, \mathrm{CH}_{3}-\mathrm{C} 6\right), 1.54-1.79\left(2 \mathrm{H}, \mathrm{m}, \mathrm{CH}_{2} \mathrm{C}_{2} \mathrm{CH}_{2}\right), 2.14(2 \mathrm{H}, \mathrm{m}$, $\left.\mathrm{CH}_{2} \mathrm{P}\right), 2.68-3.02(1 \mathrm{H}, \mathrm{m}, \mathrm{C} \underline{\mathrm{H} H N})$ 3.17-3.32 (2H, m, CㅍHN and $\left.\mathrm{MeC} \underline{\mathrm{HPh}}\right), 3.71\left(1 \mathrm{H}, \mathrm{dd}, \mathrm{J}_{\mathrm{H}}=\right.$ $\left.15 \mathrm{~Hz}, \mathrm{~J}_{\mathrm{P}}=9 \mathrm{~Hz}, \mathrm{PhC} \underline{\mathrm{H}}\right), 4.03\left(1 \mathrm{H}, \mathrm{dd}, \mathrm{J}_{\mathrm{H}}=15 \mathrm{~Hz}, \mathrm{~J}_{\mathrm{P}}=6 \mathrm{~Hz}, \mathrm{PhC} \underline{H} H\right), 4.46(1 \mathrm{H}, \mathrm{m}$, $\mathrm{C} \underline{\mathrm{HMeO}}), 7.17-7.31(10 \mathrm{H}, \mathrm{m}$, aromatic $\mathrm{H}) .{ }^{31} \mathrm{P}\{\mathrm{H}\} \mathrm{NMR}\left(\mathrm{CDCl}_{3}\right): \delta 31.60 .{ }^{13} \mathrm{C}\{\mathrm{H}\} \mathrm{NMR}$ $\left(\mathrm{CDCl}_{3}\right): \delta 22.08\left(\mathrm{~d}, \mathrm{~J}_{\mathrm{P}}=8 \mathrm{~Hz}, \underline{\mathrm{CH}}_{3} \mathrm{CHPh}\right), 24.41\left(\mathrm{~d}, \mathrm{~J}_{\mathrm{P}}=10 \mathrm{~Hz}, \underline{\mathrm{CH}}_{3}-\mathrm{C} 6\right), 32.84$ (d, J $=3 \mathrm{~Hz}$, C-5), $35.31\left(\mathrm{~d}, \mathrm{~J}_{\mathrm{P}}=3 \mathrm{~Hz}, \mathrm{Me} \underline{\mathrm{CHPh}}\right), 37.26\left(\mathrm{~d}, \mathrm{~J}_{\mathrm{P}}=131 \mathrm{~Hz}, \underline{\mathrm{CH}_{2} \mathrm{P}}\right), 44.20\left(\mathrm{CH}_{2} \mathrm{~N}\right), 51.22\left(\mathrm{~d}, \mathrm{~J}_{\mathrm{P}}=\right.$ $4 \mathrm{~Hz}, \mathrm{CH}_{2} \mathrm{Ph}$ ), 72.45 (d, $\mathrm{J}_{\mathrm{P}}=7 \mathrm{~Hz}, \underline{\mathrm{CHMeO}}$ ), 126.28 (aromatic $\mathrm{CH}$ ), 126.78 (aromatic $\mathrm{CH}$ ), 127.36 (aromatic $\mathrm{CH}$ ), 128.25 (aromatic $\mathrm{CH}$ ), 128.47 (aromatic $\mathrm{CH}$ ), 128.52 (aromatic $\mathrm{CH}$ ), $137.66\left(\mathrm{~d}, \mathrm{~J}_{\mathrm{P}}=5 \mathrm{~Hz}\right.$, aromatic $\left.\mathrm{C}\right), 147.10\left(\mathrm{~d}, \mathrm{~J}_{\mathrm{P}}=10 \mathrm{~Hz}\right.$, aromatic $\left.\mathrm{C}\right) ; \mathrm{m} / \mathrm{z}(\mathrm{EI}) 343\left(\mathrm{MH}^{+}, 14\right)$, 252 (100), 225 (90); HRMS calc for $\mathrm{C}_{20} \mathrm{H}_{26} \mathrm{NO}_{2} \mathrm{P}\left(\mathrm{MH}^{+}\right)$343.1701, found 343.1664.

\section{Acknowledgement}

We would like to thank King's College London for studentship to EDP.

\section{References}

1. Mitchell, H. J.; Nelson, A.; Warren, S. J. Chem. Soc., Perkin Trans. 1 1999, 1899.

2. Afarinkia, K.; Binch, H. M.; De Pascale, E. Synlett 2000, 1769.

3. Afarinkia, K.; Binch, H. M., Forristal, I. Synlett 2000, 1771.

4. Afarinkia, K.; De Pascale, E. Synlett 2002, 990.

5. Afarinkia, K.; Binch, H. M.; Modi, C. Tetrahedron Lett. 1998, $39,7419$.

6. Seebach, D.; Beck, A. K.; Breitschuh, R.; Job, K. Org. Synth. 1992, 71, 39. 